\title{
The Impact of User Behavior on Information Diffusion in D2D Communications: A Discrete Dynamical Model
}

\author{
Chenquan Gan (iD), Xiaoke Li (iD), Lisha Wang, and Zufan Zhang $(\mathbb{D}$ \\ School of Communication and Information Engineering, Chongqing University of Posts and Telecommunications, \\ Chongqing 400065, China \\ Correspondence should be addressed to Xiaoke Li; lxkever@foxmail.com
}

Received 6 September 2018; Revised 23 November 2018; Accepted 26 November 2018; Published 9 December 2018

Academic Editor: Guang Zhang

Copyright (c) 2018 Chenquan Gan et al. This is an open access article distributed under the Creative Commons Attribution License, which permits unrestricted use, distribution, and reproduction in any medium, provided the original work is properly cited.

This paper aims to explore the impact of user behavior on information diffusion in D2D (Device-to-Device) communications. A discrete dynamical model, which combines network metrics and user behaviors, including social relationship, user influence, and interest, is proposed and analyzed. Specifically, combined with social tie and user interest, the success rate of data dissemination between D2D users is described, and the interaction factor, user influence, and stability factor are also defined. Furthermore, the state transition process of user is depicted by a discrete-time Markov chain, and global stability analysis of the proposed model is also performed. Finally, some experiments are examined to illustrate the main results and effectiveness of the proposed model.

\section{Introduction}

The fast development of communication technologies, enhanced devices, and multimedia services will lead to a drastic change in the way of perceiving and interacting with the world around us [1]. The emerging 5G systems would transform current reality into a "connected reality", in which objects and people are interconnected in a unified whole [2]. To realize this vision, the Long Term Evolution (LTE) radio technology is adopted as a lifeline for reality scenarios. As a result, future $5 \mathrm{G}$ systems require novel approaches in terms of network design and information dissemination and should provide low latencies and timely connectivity in case of information sharing such as disasters, concerts, and other timely situations [3].

As a key component of 5G systems, D2D communications are proposed as an effective paradigm to reduce information diffusion time [4]. Reference [5] provided a game theoretical formulation for energy minimization in cooperative networks, in which the Nash bargaining solution is derived to achieve a tradeoff between energy reduction and fairness. Indeed, the direct communication between two adjacent user devices is regarded as a promising technology in future $5 \mathrm{G}$ systems, which has advantages of coverage extension, high spectrum utilization, and low energy consumption $[6,7]$.
Obviously, when data must be sent to a group of users through reliable and low latency links, D2D communications are useful for information dissemination. Consequently, the study of information diffusion is of great significance in $5 \mathrm{G}$ systems.

In $\mathrm{D} 2 \mathrm{D}$ communications, social network is an important carrier of information [8,9]. Some previous work exploited social metrics to improve information dissemination performance. For instance, $[10,11]$ gave some extensive analysis of information diffusion approaches, and different quantification models with social relationship were proposed [12-14]. Additionally, Zhang et al. [15] offloaded social network traffic through D2D links to improve the content dissemination time. With consideration of content interests and social network relations, [16] designed a social-aware data sharing strategy, whose effectiveness and functionality is verified through experimental analysis.

In social network, behavior characteristics of different users are crucial factors for information dissemination. Combined with proximity-based communication, rental work investigated the integration of communication and social domains based on user behaviors [17]. References [18, 19] exploited preference features, which indicate how much information the user needs to select seed users for sending information. Furthermore, user selfishness directly affected 


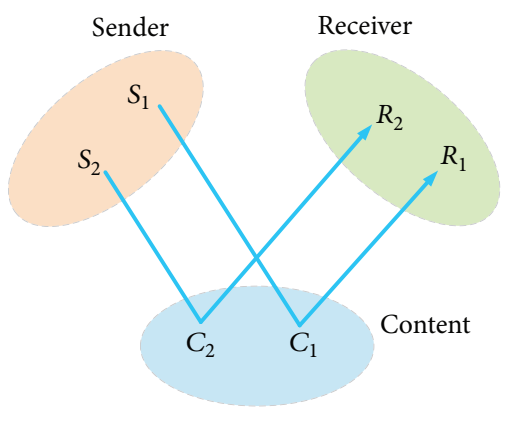

FIGURE 1: Relationship of three entities in information diffusion process.

the share willingness and the success rate of establishing D2D links [20-22]. Wang et al. [23] constructed a traffic offloading framework, in which a group of user devices were chosen as seeds based on their social influence. In [24], authors focused on maximizing cellular traffic offloading with D2D communications via content caching and users pairing. Reference [25] exploited the social tie and influence among individuals, which is modeled by Indian Buffet Process, to improve the performance and reduce the load on the wireless cellular system. Although the above-mentioned information diffusion approaches provide high data-rate and low latency, they cannot adequately characterize social interaction among users. Besides, they almost only describe the one-hop dissemination so that they fail to characterize the whole diffusion process in the network. In reality, information diffusion is a dynamic process, and its performance is not only affected by the network architecture, but by the social metrics of user as well.

Information diffusion process can be decomposed into multiple end-to-end information transmission cases; each case involves three entities, namely, sender, receiver, and content (see Figure 1). The impact factors of information diffusion include not only the attribute of sender and receiver, but also the content characteristic of information and the mutual relationship between users [26-28]. To overcome the defect of conventional models and inspired by the idea of epidemic models (e.g., [29-34]), this paper proposes a susceptible-infected-recovered (SIR) information diffusion model, which combines network metrics and user behaviors, including social relationship between users, user influence, and interest. Particularly, with consideration of social relationship and user interest, the success rate of data dissemination between D2D users is described, and the interaction factor, user influence, and stability factor are also defined. Additionally, combined with the discrete-time Markov chain, the state transition equation is established to describe the information diffusion process. Finally, global stability analysis and some experiments of the proposed model are also performed.

The subsequent materials of this paper are organized as follows: Section 2 describes mathematical framework. Model analysis and some experiments are examined in Sections 3 and 4, respectively. Finally, Section 5 outlines this work.

\section{Mathematical Framework}

In this paper, let a graph $G=(V, E)$ with $N>0$ nodes represent the information propagation network, where $V$ and $E$ denote the set of users and communication links between users, respectively. At any time, a node is in one of the following states.:

(i) $S$ (susceptible): the user who does not receive the information.

(ii) $I$ (infected): the user who has received the information and forwarded it.

(iii) $R$ (recovered): the user who has received the information but never forwards it.

\subsection{Notations}

(i) inf $f_{i}^{t}$ : the influence degree of user $i$ at time $t$, it is effected by the user's relative weight in its neighbors.

(ii) $h_{i j}$ : the success rate of data dissemination that user $i$ sends to user $j$, whose impact factors include the interest degree, social tie of users and the physical structure of network.

(iii) $\Phi_{i j}$ : the social tie strength between users $i$ and $j$, that indirectly characterize the link quality between users.

(iv) $r_{j}$ : the interest degree of user $j$ for the information.

(v) $p_{i}^{t}(X)$ : the probability that user $i$ is in state $X$ at time $t, X \in\{S, I, R\}$.

(vi) $p_{X Y}^{i}(t)$ : the transition probability of user $i$ from state $X$ to state $Y$ at time $t, X, Y \in\{S, I, R\}$.

(vii) $a_{i j}$ : the element of adjacent matrix indicating whether users $i$ and $j$ are adjacent.

(viii) $I F(i, j)$ : the interaction factor of social tie between users $i$ and $j$, referring to the historical interaction in a certain interval.

(ix) $s f(i, j)$ : the stability factor that manifests the numeric fluctuation of social tie between users $i$ and $j$.

\subsection{Model Assumptions}

(A1) Only within the scope of D2D communications can users establish communication links to transmit information.

(A2) Considering the interaction behavior which reflects the social relationship between users, and the latter determines the former in turn, an interaction factor is introduced to partly characterize the social relationship. Interaction factor reflects the subjective aspects to establish trustworthy feelings or experiences based on users' historical interactions, and each interaction is judged by a numeric degree to signify the opinion of users. Then,

$$
\operatorname{IF}(i, j)=\frac{\sum_{k=1}^{n} a c t(i, j)_{k}}{n} \times\left(1-\frac{m}{n}\right)^{1 /(n-m)},
$$


where $\operatorname{act}(i, j)_{k} \in[0,1]$ represents the evaluation value of social relationship for the $k$-th interaction between users $i$ and $j$ and $n$ and $m$ are the numbers of total and negative interactions, respectively.

Obviously, $\operatorname{IF}(i, j)$ is the accumulation of historical interactions in a certain time interval and is affected by the number of total negative judgments. The social tie strength will be weakened with the increase of negative judgments. Furthermore, the interaction is bidirectional, so $\operatorname{IF}(i, j)=\operatorname{IF}(j, i)$.

(A3) Stability factor manifests the numeric fluctuation of social tie between users. Then, it can be calculated based on the time slice aggregation as follows:

$s f(i, j)=1-\sqrt{\frac{\sum_{k=1}^{n}\left[\operatorname{Str}(i, j)^{T(k)}-\overline{\operatorname{Str}}(i, j)\right]^{2}}{n}}$,

where $T(k)$ denotes the $k$-th time slice, $\operatorname{Str}(i, j)^{T(k)}$ is the social tie strength from $i$ to $j$ in the $k$-th time slice, and $\overline{\operatorname{Str}}(i, j)$ indicates the average strength of social tie in all time slices.

The reason for considering the interaction factor and stability factor is to adjust the scenario of this paper. In particular, information transmission over physical link requires reliability and stability to guarantee the successful rate. If the social tie between users is unstable, then the infection probability is accordingly decreased, which will lead to the failure of information transmission. On the contrary, strengthened and stable social tie indicates a reliable interaction and link status, resulting in an improvement of transmission efficiency.

(A4) The social tie strength $\omega_{i j}$ is jointly determined by the interaction and stability factors. Then, $\omega_{i j}=\operatorname{IF}(i, j) *$ $s f(i, j)$.

(A5) The success rate of data dissemination is proportional to the social tie strength and interest degree of user. Then, it can be expressed as

$$
h_{i j}=\left(\beta \omega_{i j}+\gamma r_{j}\right) a_{i j}, \quad i, j=1,2, \ldots, N \text {, }
$$

where $\beta, \gamma>0$ can be adjusted according to the actual scenario.

(A6) The state transition probability from $I$ to $R$ is determined by the user's spreading ability. A stronger spreading ability corresponds to a longer survival time of information, and this time length is positively correlated to the user influence.

Based on the fact that user influence decays over time and inspired by attenuation models $[35,36]$, especially the exponential model with a long tail characteristic, the influence of user $i$ at time $t$ is defined as

$$
i n f_{i}^{t}=i n f_{i}^{t_{1}} * e^{-\left(t-t_{1}\right)}
$$

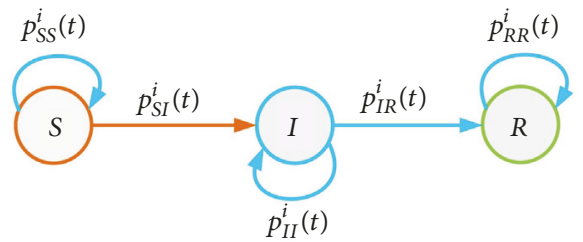

FIGURE 2: The state transition diagram of user $i$ at time $t$.

where

$$
i n f_{i}^{t_{1}}=\min \left\{\frac{M(i)}{\sum_{j \in \Gamma(i)} M(j) / M(i)}, 1\right\},
$$

$t_{1}$ denotes the initial time, $\Gamma(i)$ is the set of neighbors of user $i$, and $M(\cdot)$ is the number of user neighbors.

2.3. Model Formulation. Before giving the mathematical expression of the model, let us first introduce some basic information diffusion rules.

(R1) If a node is in state $S$, it can only receive information from its neighbors at each moment, and once it receives the information, it will transform into state $I$ and never receive the same information from other neighbors. Then, $p_{S S}^{i}(t)+p_{S I}^{i}(t)=1$, and $p_{S I}^{i}(t)=$ $1-\prod_{j \in \Gamma_{i}}\left(1-h_{i j} p_{j}^{t}(I)\right)$.

(R2) If a node is in state $I$, it will forward the information according to its influence, and then will covert to state $R$ after the survival time of information.

(R3) If a node is in state $R$, it will always maintain state $R$ and will no longer receive and forward the same information. Then, $p_{R R}^{i}(t)=1$.

Collecting the foregoing assumptions and information diffusion rules, the state transition diagram of user $i$ at time $t$ can be represented either by Figure 2 or the discrete-time Markov chain:

$$
\begin{aligned}
& p_{i}^{t+1}(S)=p_{i}^{t}(S) p_{S S}^{i}(t), \\
& p_{i}^{t+1}(I)=p_{i}^{t}(I) p_{I I}^{i}(t)+p_{i}^{t}(S) p_{S I}^{i}(t), \\
& p_{i}^{t+1}(R)=p_{i}^{t}(R) p_{R R}^{i}(t)+p_{i}^{t}(I) p_{I R}^{i}(t), \\
& p_{i}^{t}(S)+p_{i}^{t}(I)+p_{i}^{t}(R)=1,
\end{aligned}
$$

\section{Model Analysis}

The equilibrium and its stability of system (6) will be introduced in this section.

Theorem 1. Assume that $E\left(p_{i}^{*}(S), p_{i}^{*}(I), p_{i}^{*}(R)\right), i=1,2$, $\ldots, N$, is the equilibrium of system (6). Then, $p_{i}^{*}(S)=p_{i}^{*}(I)=$ 0 , and $p_{i}^{*}(R)=1$. 
Proof. According to the definition of equilibrium, one can get

$$
\begin{aligned}
& p_{i}^{*}(S)=p_{i}^{*}(S) p_{S S}^{i}(t), \\
& p_{i}^{*}(I)=p_{i}^{*}(I) p_{I I}^{i}(t)+p_{i}^{*}(S) p_{S I}^{i}(t), \\
& p_{i}^{*}(R)=p_{i}^{*}(R) p_{R R}^{i}(t)+p_{i}^{*}(I) p_{I R}^{i}(t), \\
& p_{i}^{*}(S)+p_{i}^{*}(I)+p_{i}^{*}(R)=1,
\end{aligned}
$$

$$
i=1,2, \ldots, N \text {. }
$$

Solving system (7), one can obtain $p_{i}^{*}(S)=p_{i}^{*}(I)=0$, and $p_{i}^{*}(R)=1$. Thus, the claimed result follows.

From assumption (A6) and rule (R2), the probability that user stays in state $I$ is positively correlated to its influence. So one might as well make $p_{I I}^{i}(t)=i n f_{i}^{t}$. Then, $p_{I R}^{i}(t)=1-p_{I I}^{i}(t)$, and system (6) can be rewritten as

$$
\begin{aligned}
& p_{i}^{t+1}(I) \\
& \quad=\inf f_{i}^{t} p_{i}^{t}(I) \\
& \quad+\left(1-\prod_{j \in \Gamma_{i}}\left(1-h_{i j} p_{j}^{t}(I)\right)\right)\left(1-p_{i}^{t}(I)-p_{i}^{t}(R)\right), \\
& p_{i}^{t+1}(R)=p_{i}^{t}(R)+\left(1-i n f_{i}^{t}\right) p_{i}^{t}(I), \quad \\
& \quad i=1,2, \ldots, N .
\end{aligned}
$$

Note that

$$
\begin{aligned}
p_{i}^{t+1}(I) & \\
= & \inf _{i}^{t} p_{i}^{t}(I) \\
& +\left(1-\prod_{j \in \Gamma_{i}}\left(1-h_{i j} p_{j}^{t}(I)\right)\right)\left(1-p_{i}^{t}(I)-p_{i}^{t}(R)\right) \\
& \leq \inf _{i}^{t} p_{i}^{t}(I)+\left(1-p_{i}^{t}(I)-p_{i}^{t}(R)\right) \sum_{j \in \Gamma(i)} h_{i j} p_{j}^{t}(I) \\
\leq & \inf _{i}^{t} p_{i}^{t}(I)+\sum_{j \in \Gamma(i)} h_{i j} p_{j}^{t}(I) .
\end{aligned}
$$

Hence, it suffices to consider the following system:

$$
\begin{aligned}
& p_{i}^{t+1}(I)=i n f_{i}^{t} p_{i}^{t}(I)+\sum_{j \in \Gamma(i)} h_{i j} p_{j}^{t}(I), \\
& p_{i}^{t+1}(R)=p_{i}^{t}(R)+\left(1-i n f_{i}^{t}\right) p_{i}^{t}(I),
\end{aligned}
$$

$$
i=1,2, \ldots, N \text {. }
$$

Besides, systems (10) and (8) have the same equilibrium $E\left(p_{i}^{*}(I)=0, p_{i}^{*}(R)=1\right), i=1,2, \ldots, N$.
Define

$$
\begin{aligned}
\mathbf{p}^{t}(\cdot) & =\left(p_{1}^{t}(\cdot), p_{2}^{t}(\cdot), \ldots, p_{N}^{t}(\cdot)\right)^{T}, \\
\mathbf{I}_{N} & =\left(\begin{array}{cccc}
1 & 0 & \cdots & 0 \\
0 & 1 & \cdots & 0 \\
\vdots & \vdots & \vdots & \vdots \\
0 & 0 & \cdots & 1
\end{array}\right), \\
\mathbf{H} & =\left(\begin{array}{cccc}
h_{11} & h_{12} & \cdots & h_{1 N} \\
h_{21} & h_{22} & \cdots & h_{2 N} \\
\vdots & \vdots & \vdots & \vdots \\
h_{N 1} & h_{N 2} & \cdots & h_{N N}
\end{array}\right), \\
\operatorname{Inf}_{N} & =\left(\begin{array}{cccc}
i n f_{1}^{t} & 0 & \cdots & 0 \\
0 & i n f_{2}^{t} & \cdots & 0 \\
\vdots & \vdots & \vdots & \vdots \\
0 & 0 & \cdots & \text { inf }_{N}^{t}
\end{array}\right) .
\end{aligned}
$$

Then, $\mathbf{p}^{t+1}(I)=\left(\mathbf{H}+\mathbf{I n f}_{N}\right) \mathbf{p}^{t}(I)$. It follows from stability theory of autonomous linear systems [37] that the equilibrium of system (10) is globally asymptotically stable if $\lambda_{\max }(\mathbf{H}+$ Inf $\left._{N}\right)<1$.

Now, the main result of this paper can be derived as follows.

Theorem 2. The equilibrium $E\left(p_{i}^{*}(S), p_{i}^{*}(I), p_{i}^{*}(R)\right), i=$ $1,2, \ldots, N$, of system (6) is globally asymptotically stable if $\lambda_{\max }\left(\mathbf{H}+\mathbf{I n f}_{N}\right)<1$.

Remark 3. At the stable point, the number of users that have received information (users in state $R$ ) represents the transmission range. That is to say, a larger number of users in state correspond to a wider coverage of information. Obviously, the transmission range is affected by the interest degree, social tie, and other factors, which is reasonable in the actual situation.

\section{Experiments}

To validate the effectiveness of the proposed model, this section simulates the information diffusion process with MATLAB in a random network, in which each node represents a user. At each time point, users take the receiving or forwarding decision according to its own state and the system parameters. Meanwhile, the impact of user influence and information popularity on information diffusion is also illustrated. Here, let us introduce two important indexes in advance.

Max Infection Peak (MIP). MIP refers to the ratio of the maximum number of infected users to the total number of users. This index characterizes the information diffusion speed, and a larger MIP means a wider coverage of information. 


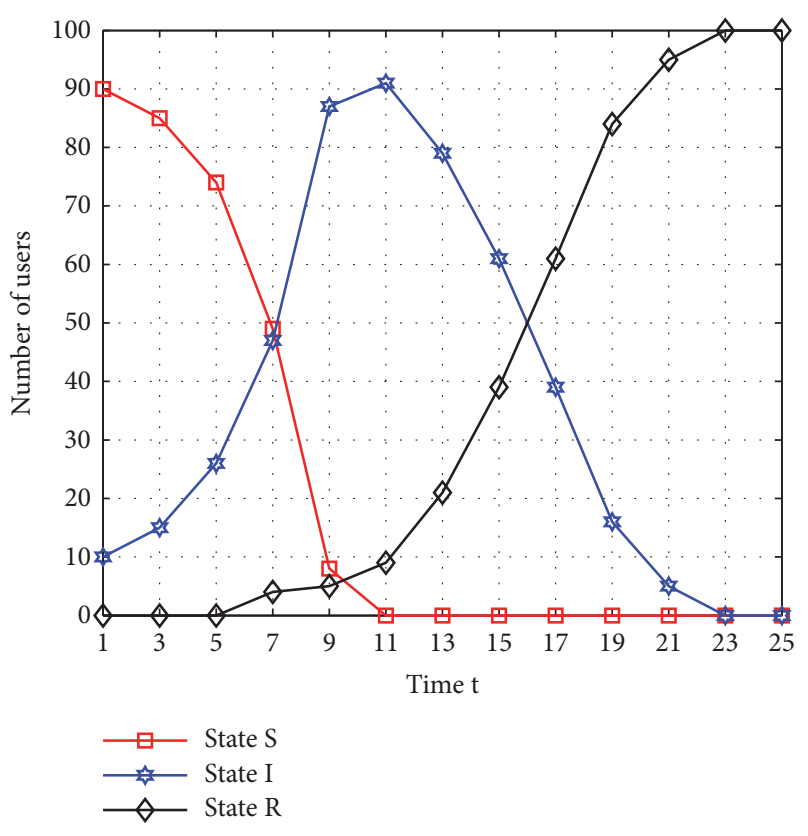

FIGURE 3: System convergence process.

Average Influence $(A I N)$. AIN is the average influence of all users in the network, and the influence degree of each user is derived from (4).

Example 4. Consider system (8) with system parameters $b=0.6, y=0.4$. Meanwhile, take a network with 100 total nodes and 10 seed nodes as the propagation network. Figures 3, 4, 5, 6 , and 9 show the model analysis under this condition.

Figure 3 presents the number of users in each state over time. It can be seen that the total number of users at each time point is equal to 100 , and the user number in state $R$ is zero at initial time. This is because only the seed users hold the information at initial time, so other users are in state $S$. Meanwhile, the system converges to a stable state, which is consistent with the theory analysis (i.e., Theorems 1 and 2), and the convergence speed is determined by some parameters, such as the network topology (the adjacent matrix), social tie, and user influence.

Figure 4 illustrates the users state in different time points at $\mathrm{t}=2,10,18$, respectively, in which the red, blue, and black circles indicate users in S, I, R state, respectively. It is obvious that the varying number of users in each state of (a), (b), and (c) is consistent with the theoretical analysis.

Figure 5 compares the percentage of each state user between awareness and no awareness. Figures 5(a), 5(b), and 5(c) show the number of susceptible, infected, and recovered users, respectively. It can be observed that the percentage of infected user under the proposed model is larger than that of the model without consideration of social attribute of users and information. This is because the proposed model considers the social tie and user influence, resulting in the fact that information is transmitted with a relative higher successful rate and a wider coverage as shown in Figure 5(c).
In addition, it can be found that the model without awareness has higher users in state R over a major part of the time scale. This is because the diffusion model without awareness ignores the impact of influential users, so that the recovery rate is faster than that of the proposed model.

Figure 6 displays the impact of user influence on the number of users in each state. By comparing Figures 6(a), $6(\mathrm{~b})$, and $6(\mathrm{c})$, it can be concluded that the rate of user transforming from state $S$ to $I$ increases with the AIN of user. The reason is that the average influence of users is larger, and the interaction between users will be stronger, so that user is more likely to become infected. Meanwhile, user influence is closely associated with the survival time of information, and a larger $A I N$ can extend the length of this time. Therefore, more influential user can expand the diffusion range of information. In addition, with the increase of user influence, the time point that users reach to peak of $I$ state is earlier, so that system converges to a stable state with a fast speed.

Example 5. Consider system (8) with system parameters $b=0.6, y=0.4$ and 10 initial seed nodes. Figures 7 and 8 illustrate the diffusion model with total nodes 60,70 , and 100 , respectively.

Figures 7 and 8 show the evolution of infected and recovered users under different destinations. With the increase of destinations, there is a slightly delay in the time that reaches to peak point of infected users. However, the time delay is quite limited although increasing the number of destinations of almost $67 \%$ (from 60 to 100). Furthermore, it should be noted that after almost users are infected, a parallel increase in the number of recovered users is met till all users become recovered, which manifests that a higher number of destinations imply a faster recovery process.

Figure 9 demonstrates a comparison of MIP between awareness and no awareness models. Obviously, the MIP increasing with the information popularity is independent of model. The reason is that the information popularity is derived from the user interest degree, and a higher interest degree corresponds to a larger popularity. That is to say, if the information is enjoyed by a higher popularity, the transmission probability will increase accordingly, leading to a greater diffusion speed. In this case, most users are in state $I$ before going to state $R$. Additionally, the MIP of the proposed model is higher than that of the model without consideration of social attribute of users. The reason is that the proposed model takes the social tie and user interest degree into account, so that the role of authority user is fully played to expand the diffusion range.

\section{Conclusions and Future Work}

In this paper, with joint consideration of social tie, user influence, and user interest, a user behavior based information diffusion model for $5 \mathrm{G}$ systems has been proposed. By characterizing the state transition probability of each user, a discrete-time Markov chain system has been formulated. Furthermore, the global stability of the system has been 


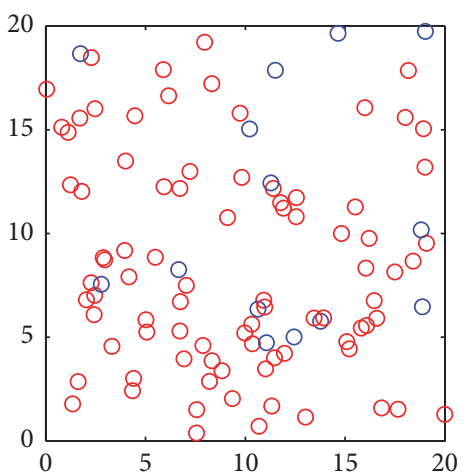

(a) $\mathrm{t}=2$

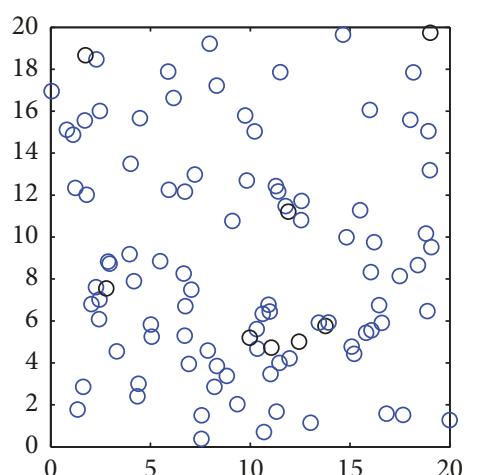

(b) $t=10$

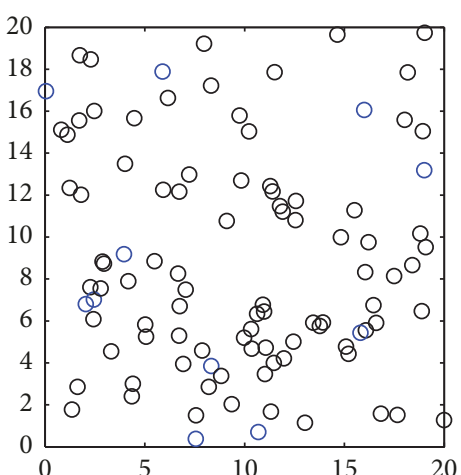

(c) $\mathrm{t}=18$

FIgURE 4: The users state in different time points at $\mathrm{t}=2,10,18$.

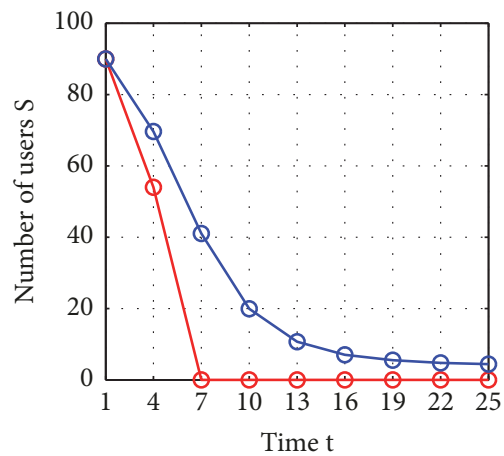

- With awareness

- Without awareness

(a)

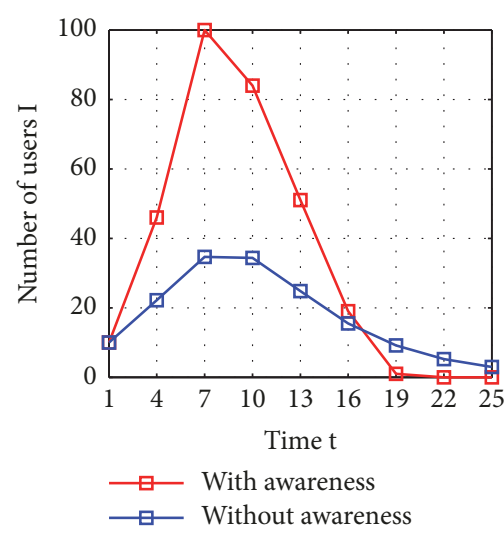

(b)

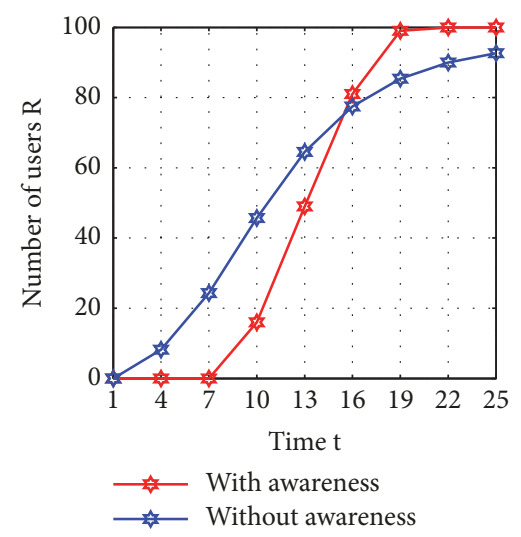

(c)

FIGURE 5: A comparison between awareness and no awareness.

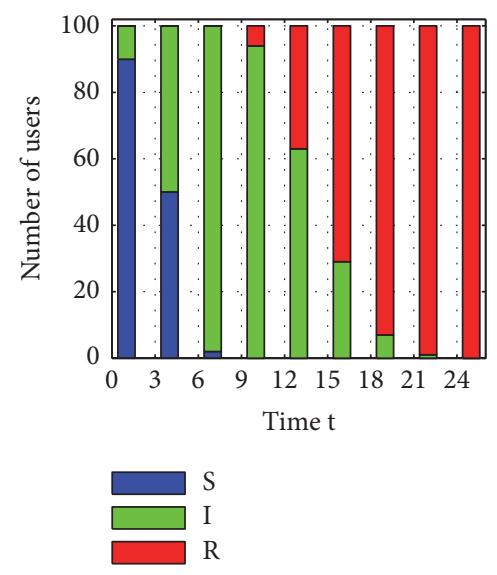

(a) $\mathrm{AIN}=0.87$

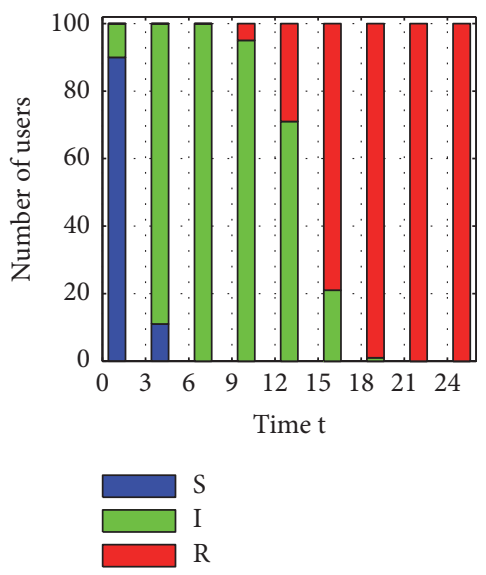

(b) AIN $=0.92$

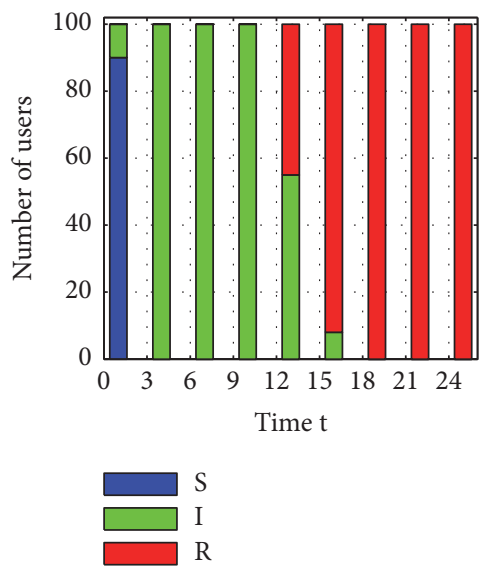

(c) $\mathrm{AIN}=0.94$

FIGURE 6: The impact of user influence on information diffusion process.

proved. Finally, some experiments have been performed to illustrate the main results and effectiveness of the proposed model.

The provided insights can guide the development of information diffusion and D2D communications. The discovery of social attributes of user and information improves the effect of information diffusion, which is relevant to advertising, public opinion monitoring, and other scenarios.

In further work, the study can be continued in several directions. On the one hand, we shall apply the theory of discrete dynamics (e.g., [37-40]) to study dynamical behavior of information diffusion and then to study the discrete 


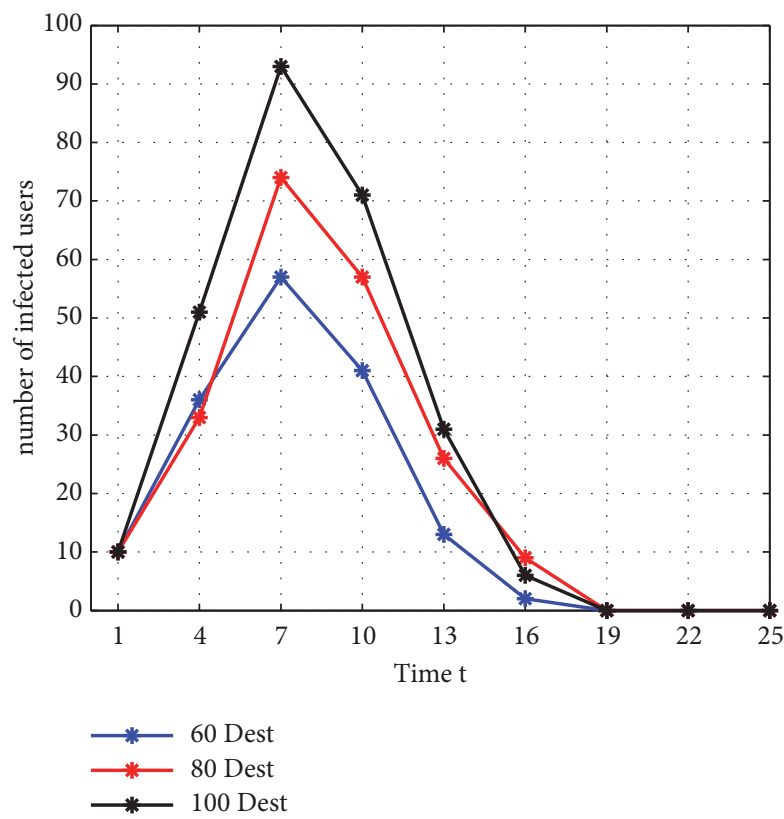

Figure 7: The evolution of infected users over time under different destinations.

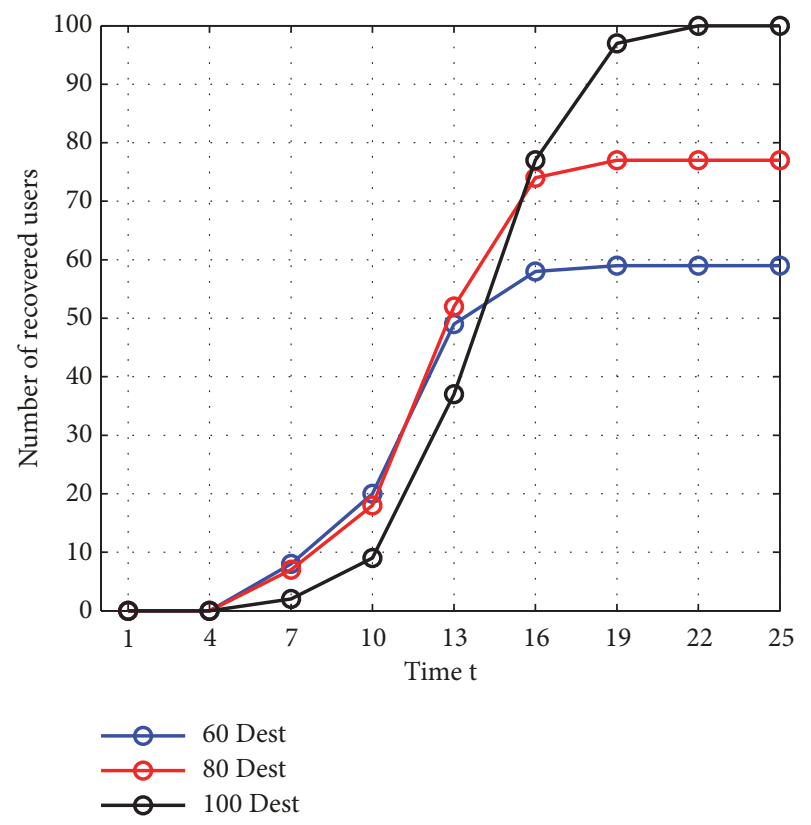

FIGURE 8: The evolution of recovered users over time under different destinations.

nonautonomous propagation model. On the other hand, the diffusion model should be improved with consideration of user sentiment analysis [41, 42], user mobility, and multitopic scenario.

\section{Data Availability}

The data used to support the findings of this study are available from the corresponding author upon request.

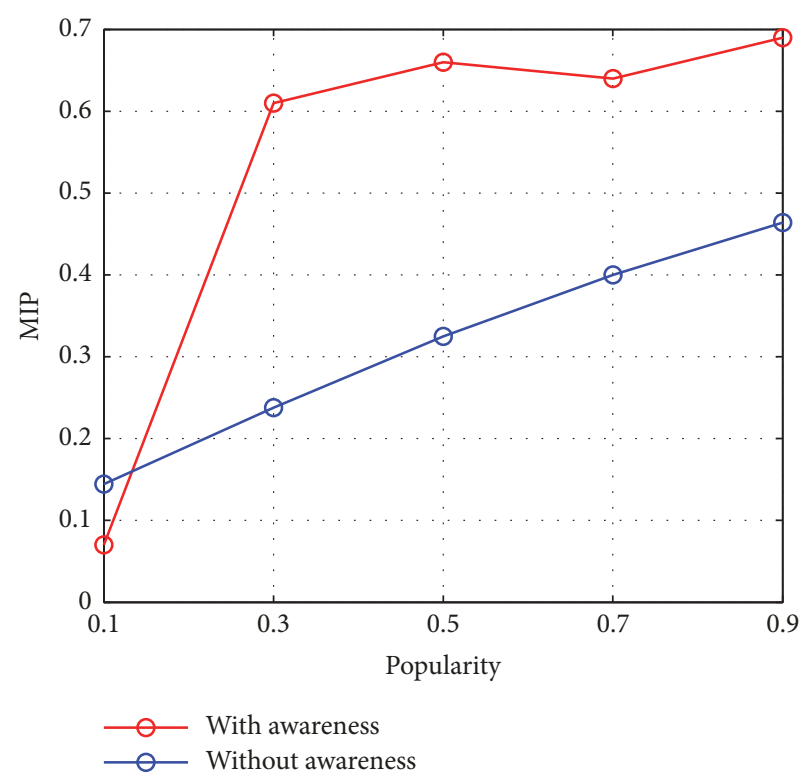

Figure 9: A comparison of MIP between awareness and no awareness models.

\section{Conflicts of Interest}

The authors declare that there are no conflicts of interest regarding the publication of this paper.

\section{Acknowledgments}

This work is supported by Natural Science Foundation of China (Grants nos. 61702066 and 11747125), Scientific and Technological Research Program of Chongqing Municipal Education Commission (Grant no. KJ1704080), Chongqing Research Program of Basic Research and Frontier Technology (Grants nos. cstc2017jcyjAX0256 and cstc2018jcyjAX0154), and Research Innovation Program for Postgraduate of Chongqing (Grants nos. CYS17217 and CYS18238).

\section{References}

[1] E. Cianca, M. De Sanctis, and M. Ruggieri, "Convergence towards 4G: A novel view of integration," Wireless Personal Communications, vol. 33, no. 3-4, pp. 327-336, 2005.

[2] I. Chih-Lin, S. Han, Y. Chen, and G. Li, "Trillions of nodes for 5G!?" in Proceedings of the 2014 IEEE/CIC International Conference on Communications in China, ICCC 2014, pp. 246250, China, October 2014.

[3] M. A. M. Albreem, "5G wireless communication systems: Vision and challenges," in Proceedings of the 2nd International Conference on Computer, Communications, and Control Technology, I4CT 2015, pp. 493-497, Malaysia, April 2015.

[4] A. Pyattaev, O. Galinina, S. Andreev, M. Katz, and Y. Koucheryavy, "Understanding practical limitations of network coding for assisted proximate communication," IEEE Journal on Selected Areas in Communications, vol. 33, no. 2, pp. 156-170, 2015.

[5] E. Yaacoub, L. Al-Kanj, Z. Dawy, S. Sharafeddine, F. Filali, and A. Abu-Dayya, "A Nash bargaining solution for energy-efficient 
content distribution over wireless networks with mobile-tomobile cooperation," in Proceedings of the 4th Joint IFIP Wireless and Mobile Networking Conference (WMNC '11), pp. 1-7, Toulouse, France, October 2011.

[6] N. Reider and G. Fodor, "A distributed power control and mode selection algorithm for D2D communications," Eurasip Journal on Wireless Communications \& Networking, vol. 1, p. 266, 2012.

[7] L. Lei, Z. D. Zhong, C. Lin, and X. M. Shen, "Operator controlled device-to-device communications in LTE-advanced networks," IEEE Wireless Communications Magazine, vol. 19, no. 3, pp. 96104, 2012.

[8] E. Bakshy, I. Rosenn, C. Marlow, and L. Adamic, "The role of social networks in information diffusion," in Proceedings of the 21st Annual Conference on World Wide Web (WWW '12), pp. 519-528, Lyon, France, April 2012.

[9] Y. Zhang, E. Pan, L. Song, W. Saad, Z. Dawy, and Z. Han, "Social network enhanced device-to-device communication underlaying cellular networks in," in proceedings of the IEEE/CIC International Conference on Communications in China Workshops, pp. 182-186, 2013.

[10] A. Guille, H. Hacid, C. Favre, and D. A. Zighed, "Information diffusion in online social networks: A survey," SIGMOD Record, vol. 42, no. 2, pp. 17-28, 2013.

[11] K. Saito, M. Kimura, K. Ohara, and H. Motoda, "Selecting Information Diffusion Models over Social Networks for Behavioral Analysis," in Proceedings of the European Conference on Machine Learning and Knowledge Discovery in Databases, vol. 6323 of Lecture Notes in Computer Science, pp. 180-195, Springer.

[12] J. Ho Choi, D. Oh Kang, J. Young Jung, and C. Bae, "Estimating Social Tie Strength for Autonomous D2D Collaborations," International Journal of Future Computer and Communication, vol. 4, no. 1, pp. 7-12, 2015.

[13] D. Wu, L. Zhou, Y. Cai, H. Chao, and Y. Qian, "Physical-SocialAware D2D Content Sharing Networks: A Provider-Demander Matching Game," IEEE Transactions on Vehicular Technology, vol. 67, no. 8, pp. 7538-7549, 2018.

[14] Y. Yi, Z. Zhang, and C. Gan, "The effect of social tie on information diffusion in complex networks," Physica A: Statistical Mechanics and its Applications, vol. 509, pp. 783-794, 2018.

[15] Y. Zhang, E. Pan, L. Song, W. Saad, Z. Dawy, and Z. Han, "Social network aware device-to-device communication in wireless networks," IEEE Transactions on Wireless Communications, vol. 14, no. 1, pp. 177-190, 2015.

[16] L. Aoude, Z. Dawy, S. Sharafeddine, K. Frenn, and K. Jahed, "Social-Aware Device-to-Device Offloading Based on Experimental Mobility and Content Similarity Models," Wireless Communications and Mobile Computing, vol. 2018, Article ID 5606829, 16 pages, 2018.

[17] Y. Li, T. Wu, P. Hui, D. Jin, and S. Chen, "Social-aware D2D communications: qualitative insights and quantitative analysis," IEEE Communications Magazine, vol. 52, no. 6, pp. 150-158, 2014.

[18] Y. Li, M. Qian, D. Jin, P. Hui, Z. Wang, and S. Chen, "Multiple mobile data offloading through disruption tolerant networks," IEEE Transactions on Mobile Computing, vol. 13, no. 7, pp. 15791596, 2014.

[19] H.-H. Cheng and K. C.-J. Lin, "Source selection and content dissemination for preference-aware traffic offloading," IEEE Transactions on Parallel and Distributed Systems, vol. 26, no. 11, pp. 3160-3174, 2015.

[20] T. Wang, Y. Sun, L. Song, and Z. Han, "Social data offloading in D2D-enhanced cellular networks by network formation games,"
IEEE Transactions on Wireless Communications, vol. 14, no. 12, pp. 7004-7015, 2015.

[21] J. Li, Q. Yang, P. Gong, and K. S. Kwak, "End-to-End Multiservice Delivery in Selfish Wireless Networks under Distributed Node-Selfishness Management," IEEE Transactions on Communications, vol. 64, no. 3, pp. 1132-1142, 2016.

[22] C. Wang, X.-Y. Yang, K. Xu, and J.-F. Ma, "SEIR-based model for the information spreading over SNS," Tien Tzu Hsueh Pao/Acta Electronica Sinica, vol. 42, no. 11, pp. 2325-2330, 2014.

[23] X. Wang, M. Chen, Z. Han, D. O. Wu, and T. T. Kwon, “TOSS: Traffic offloading by social network service-based opportunistic sharing in mobile social networks," in Proceedings of the 33rd IEEE Conference on Computer Communications, IEEE INFOCOM 2014, pp. 2346-2354, Canada, May 2014.

[24] J. Jiang, S. Zhang, B. Li, and B. Li, "Maximized cellular traffic offloading via device-to-device content sharing," IEEE Journal on Selected Areas in Communications, vol. 34, no. 1, pp. 82-91, 2016.

[25] Y. Zhang, L. Song, W. Saad, Z. Dawy, and Z. Han, "Exploring social ties for enhanced device-to-device communications in wireless networks," in Proceedings of the 2013 IEEE Global Communications Conference (GLOBECOM 2013), pp. 45974602, Atlanta, Ga, USA, December 2013.

[26] H.-F. Zhang, J.-R. Xie, M. Tang, and Y.-C. Lai, "Suppression of epidemic spreading in complex networks by local information based behavioral responses," Chaos: An Interdisciplinary Journal of Nonlinear Science, vol. 24, no. 4, 043106, 7 pages, 2014.

[27] K. Paarporn, C. Eksin, J. S. Weitz, and J. S. Shamma, "Epidemic spread over networks with agent awareness and social distancing," in Proceedings of the 53rd Annual Allerton Conference on Communication, Control, and Computing, Allerton 2015, pp. 5157, USA, October 2015.

[28] C. Lagnier, L. Denoyer, E. Gaussier, and P. Gallinari, "Predicting Information Diffusion in Social Networks Using Content and User's Profiles," in Proceedings of the 35th European Conference on IR Research, vol. 7814 of Lecture Notes in Computer Science, pp. 74-85, Springer, 2013.

[29] Q. Zhu, X. Yang, and J. Ren, "Modeling and analysis of the spread of computer virus," Communications in Nonlinear Science and Numerical Simulation, vol. 17, no. 12, pp. 5117-5124, 2012.

[30] C. Gan, X. Yang, W. Liu, Q. Zhu, and X. Zhang, "Propagation of computer virus under human intervention: a dynamical model," Discrete Dynamics in Nature and Society, vol. 2012, Article ID 106950, 8 pages, 2012.

[31] L.-X. Yang, X. Yang, J. Liu, Q. Zhu, and C. Gan, "Epidemics of computer viruses: a complex-network approach," Applied Mathematics and Computation, vol. 219, no. 16, pp. 8705-8717, 2013.

[32] C. Gan, X. Yang, W. Liu, and Q. Zhu, "A propagation model of computer virus with nonlinear vaccination probability," Communications in Nonlinear Science and Numerical Simulation, vol. 19, no. 1, pp. 92-100, 2014.

[33] W. Liu and S. Zhong, "Modeling and analyzing the dynamic spreading of epidemic malware by a network eigenvalue method," Applied Mathematical Modelling: Simulation and Computation for Engineering and Environmental Systems, vol. 63, pp. 491-507, 2018.

[34] W. Liu, X. Wu, W. Yang, X. Zhu, and S. Zhong, "Modeling cyber rumor spreading over mobile social networks: A compartment approach," Applied Mathematics and Computation, vol. 343, pp. 214-229, 2019. 
[35] J. Wallinga and P. Teunis, "Different epidemic curves for severe acute respiratory syndrome reveal similar impacts of control measures," American Journal of Epidemiology, vol. 160, no. 6, pp. 509-516, 2004.

[36] S. A. Myers and J. Leskovec, "On the convexity of latent social network inference," in Proceedings of the 23rd International Conference on Neural Information Processing Systems, pp. 17411749, 2010.

[37] R. C. Robinson, An Introduction to Dynamical Systems-Continuous and Discrete, vol. 19 of Pure and Applied Undergraduate Texts, American Mathematical Society, Providence, Second edition, 2012.

[38] C. Gan, X. Yang, and W. Liu, "On behavior of two third-order nonlinear difference equations," Utilitas Mathematica, vol. 93, pp. 193-203, 2014.

[39] C. Gan, X. Yang, and W. Liu, "Global Behavior of $\mathrm{xn}+1=(\mathrm{a}+\beta \mathrm{xn}+\mathrm{k})(\gamma+\mathrm{xn})$, Discrete dynamics in nature and society, vol. 2013, Article ID 963757, 5 pages, 2013.

[40] S. Elaydi, An Introduction to Difference Equations, Springer, New York, NY, USA, 2005.

[41] Z. Zhang, Y. Zou, and C. Gan, "Textual sentiment analysis via three different attention convolutional neural networks and cross-modality consistent regression," Neurocomputing, vol. 275, pp. 1407-1415, 2018.

[42] Z. Zhang, L. Wang, Y. Zou, and C. Gan, "The optimally designed dynamic memory networks for targeted sentiment classification," Neurocomputing, vol. 309, pp. 36-45, 2018. 


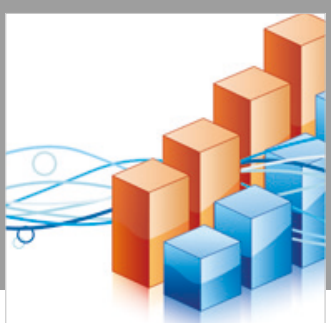

Advances in

Operations Research

\section{-n-m}
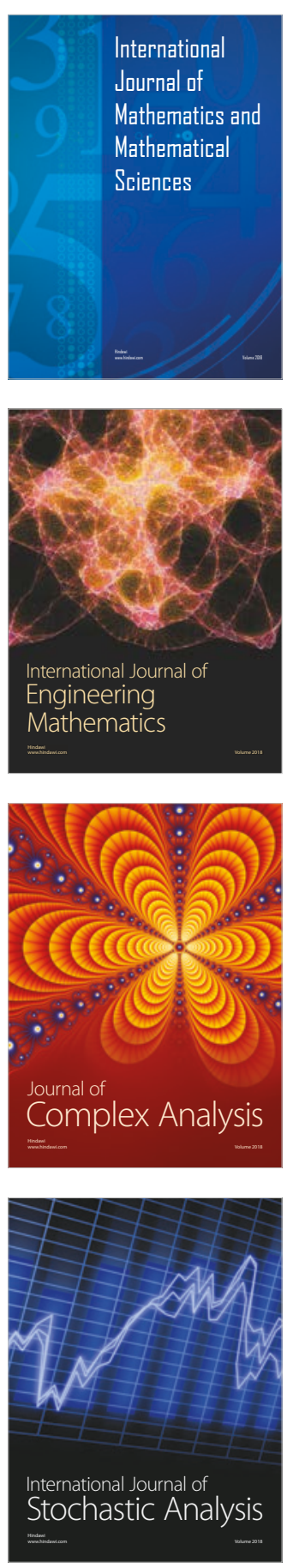
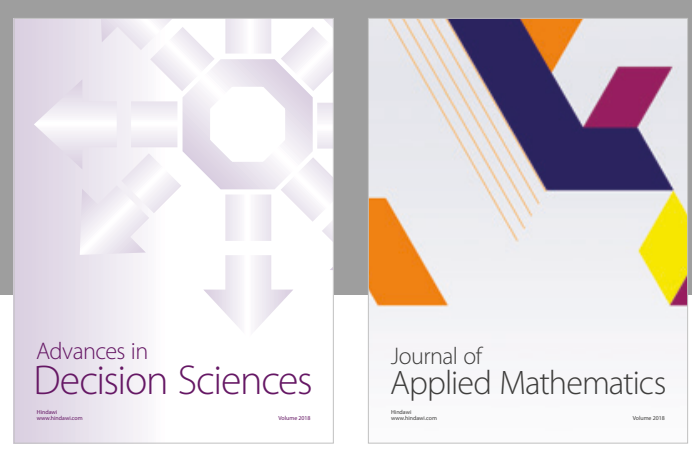

Journal of

Applied Mathematics
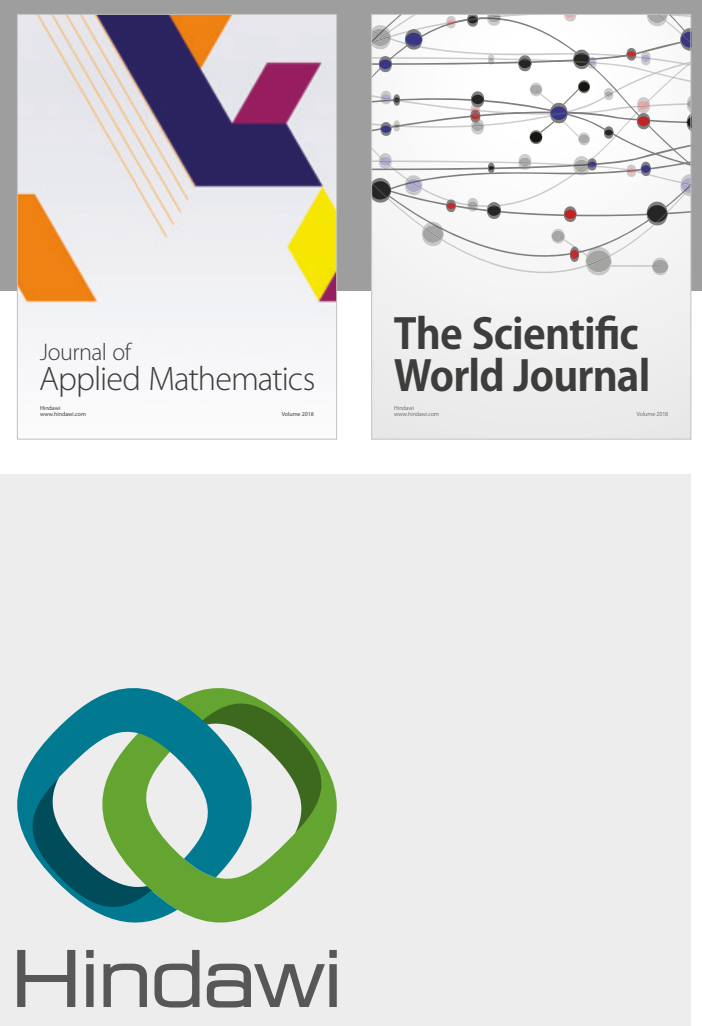

Submit your manuscripts at

www.hindawi.com

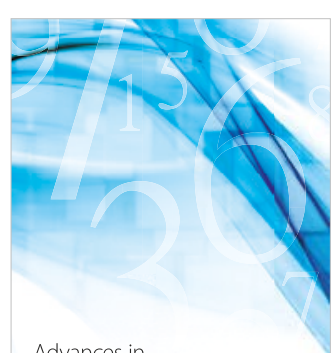

Advances in
Numerical Analysis
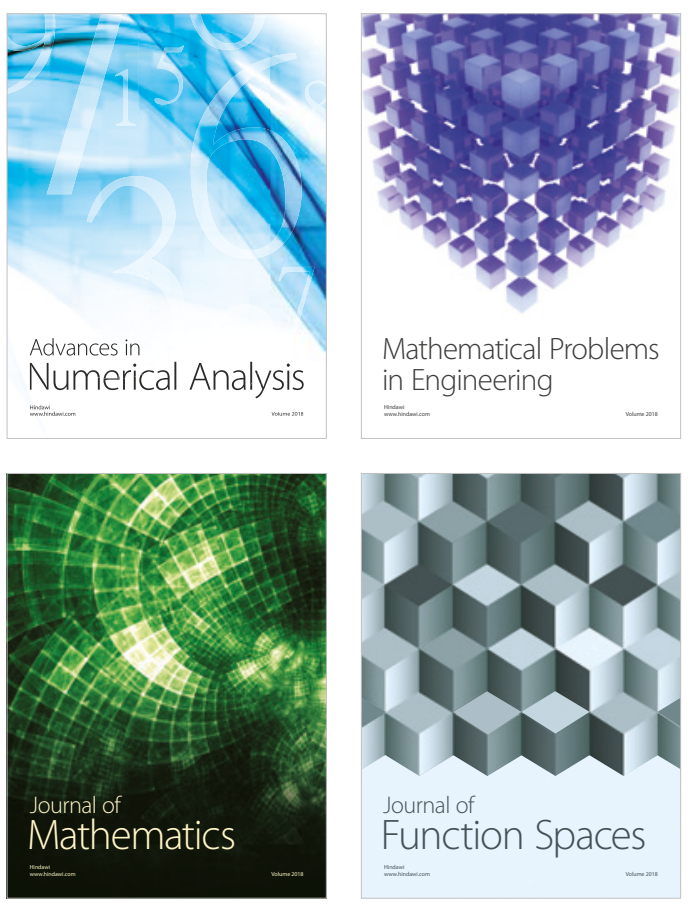

Mathematical Problems in Engineering

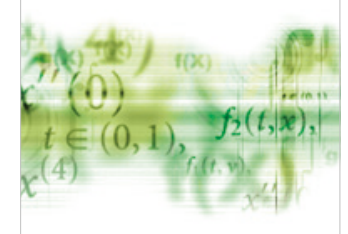

International Journal of

Differential Equations

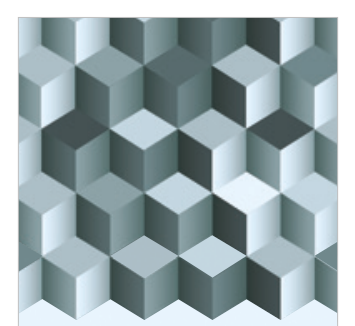

Journal of

Function Spaces

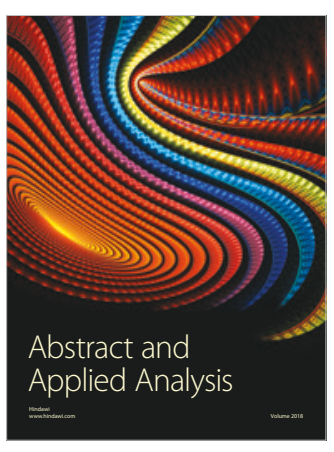

The Scientific

World Journal

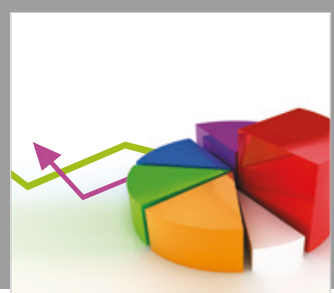

Journal of

Probability and Statistics
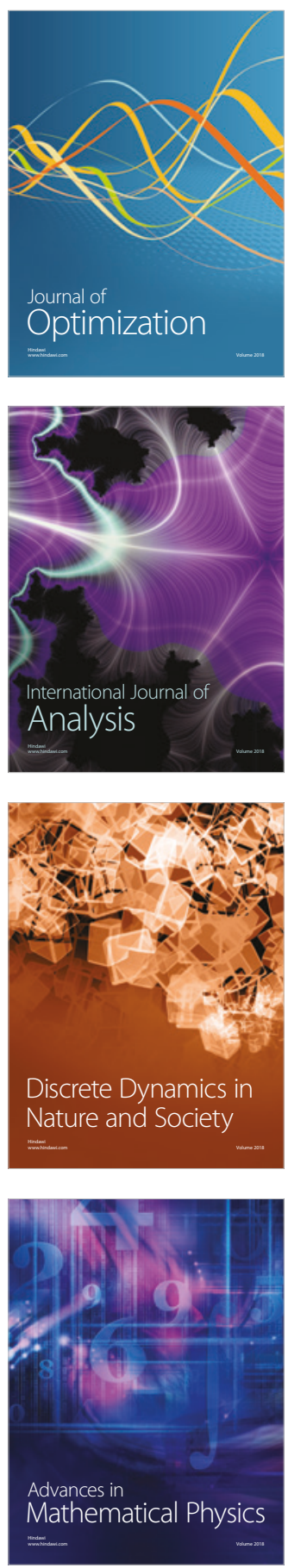